\title{
ELECTROMECHANICALLY ACTIVE FLAT OPTICAL DEVICES
}

Tapashree Roy ${ }^{1}$, 2, Shuyan Zhang ${ }^{2}$, Il Woong Jung ${ }^{l}$, Federico Capasso ${ }^{2}$, and Daniel Lopez ${ }^{\prime}$

${ }^{1}$ Nanoscience and Technology Division, Argonne National Laboratory, Lemont, IL 60439

${ }^{2}$ John A. Paulson School of Engineering and Applied Sciences, Harvard University, Cambridge, MA 02138

\section{ABSTRACT}

We demonstrate an electromechanically controllable reflective flat lens at mid-infrared wavelength. The lens is designed from plasmonic nano-discs, and focuses light at mid-infrared, $45^{\circ}$ to the incoming beam. The flat lens is fabricated on a $2.8 \mu \mathrm{m}$ thin membrane and integrated with a micro-electro-mechanical (MEMS) device. Mechanical testing of the MEMS device before and after integration of the flat lens confirms that the performance of the MEMS has not been compromised due to addition of a flat lens.

\section{INTRODUCTION}

Recently, flat optical devices capable of shaping the wavefront of light have come to the forefront of ongoing scientific research [1]. Planar counterparts of conventional optical devices like lenses [2, 3], beam deflectors [4, 5], holograms [6,7], and so on have been experimentally demonstrated. These devices, referred to as "metasurfaces", use sub-wavelength dimension metal or dielectric resonators, arbitrarily spaced on a two dimensional plane, mimicking the phase profile of any conventional bulk optical device and beyond. Such metasurface-based flat devices are compact and lightweight compared to the three-dimensional counterparts. However, most of these nanostructured devices have so far been passive; a single device provides a specific output only. For developing these flat optical elements into useful optical systems, we need to incorporate precise mechanical control of the individual elements.

In this paper we introduce a useful concept of actively controlling these flat optical devices by electrostatic actuation. We present a prototype consisting of an electromechanically controlled plasmonic flat reflective lens that focuses in the mid-infrared part of the spectrum. When electrostatically actuated, the MEMS platform controls the tilt angle of the lens along two orthogonal axes by about +- 9 degrees. In dynamic actuation the platform can be tilted to larger angles to scan the focal spot. Such actively controlled miniaturized optical devices promise to provide faster, more efficient and often enhanced functionalities.

\section{DESING OF THE LENS}

We design a plasmonic lens producing cylindrical focus when illuminated with light at wavelength of $4.58 \mu \mathrm{m}$. As the design units, we choose sub-wavelength sized gold resonators in the shape of a disc (Fig. 1a). The reflection from the gold nano-resonators is reinforced by the use of a continuous gold film, separated from the resonator by a $400 \mathrm{~nm}$ thick silicon dioxide layer. When signal is incident from the top, it interacts with the nano-disc, passes through the transparent dielectric layer and gets reflected back up from the gold backplane. The reflected signal comes out with a different amplitude and phase compared to the incident signal. By simply changing the radius of the disc, it is possible to realize different values of reflected phase between 0 and $2 \pi$ (Fig. 1b). To construct a planar lens, we spatially distribute the discs with varying radii to realize the hyperbolic phase profile on a twodimensional surface. Figure 1c shows a schematic of the reflective flat lens; light is incident normal to the lens surface, but reflects at an angle from the incoming beam, while focusing the reflected light at a predetermined distance from the lens surface. To achieve this change in path of the focused beam, we superimpose a planar phase shift on the hyperbolic phase profile. This configuration helps to dissociate the reflected light from the incident light, besides proving the flexibility of our design technique.


Figure 1: Designing the plasmonic flat lens (a) A unit cell consisting of a $50 \mathrm{~nm}$ thick gold disc on $400 \mathrm{~nm}$ thick silicon dioxide substrate with $200 \mathrm{~nm}$ thick gold backplane (b) Simulated values of reflective phase for varying sizes of gold discs. (c) Schematic representation of focusing characteristics of the reflective flat lens.

Figure 2 summarizes the simulated results for a realistic lens design at the wavelength of $4.58 \mu \mathrm{m}$. The cylindrical lens has a square layout, with each side measuring $800 \mu \mathrm{m}$. Figure 2a shows the propagation of the reflected beam along the transverse direction; the incoming beam is not shown. The lens is positioned at $y=0$, and about $x=0$. The axis of reflection makes an angle of $45^{\circ}$ to the normal of the lens, i.e. to the $y$-axis. The focal plane is $2 \mathrm{~mm}$ away from the lens surface, when measured along the axis of reflection (Fig. 2a, dotted straight line). The intensity distribution at the focal plane is shown in Fig. 2b. A focal line appears because of the cylindrical focusing characteristics of the flat lens. The spatial distribution of the phase at the plane of the lens is shown in Fig. $2 c$; the phase changes along $x$-position of the lens only.

\section{FABRICATION}

\section{The flat lens}

The flat lens is fabricated using standard photolithography techniques. As the substrate, a silicon-on-insulator (SOI) wafer is chosen with $200 \mathrm{~nm}$ buried-oxide layer and $600 \mu \mathrm{m}$ thick handle layer. Figure $3(\mathrm{a}-\mathrm{f})$ shows the process flow for fabricating the lens. In the following section we describe the fabrication steps following the figure numbers: (a) On the topside of the SOI wafer, 

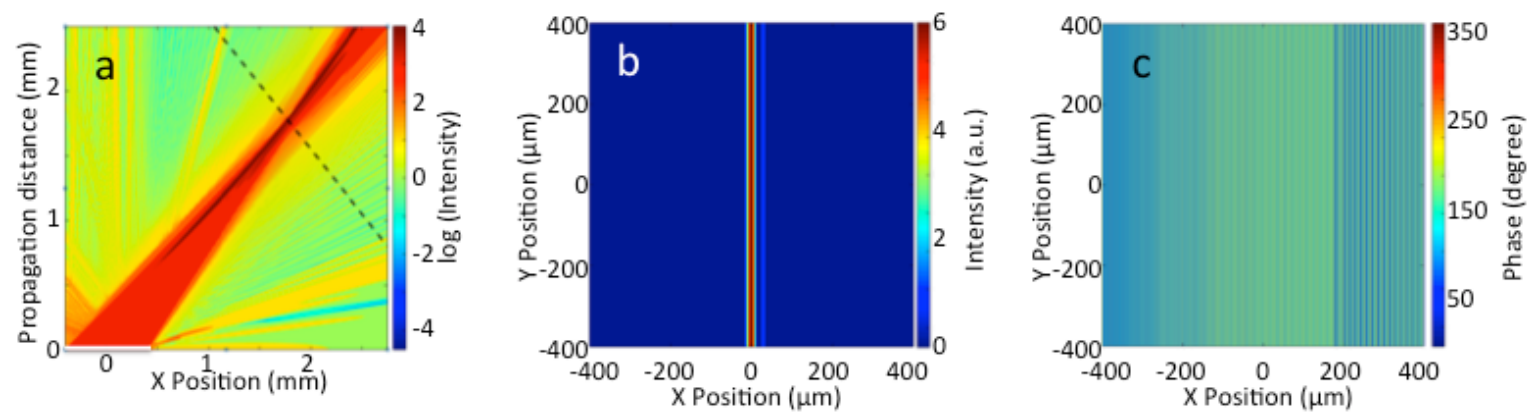

Figure 2. Simulated characteristics of a flat cylindrical lens at $\lambda=4.58 \mu \mathrm{m}$. (a) Intensity distribution of the reflected beam at the plane of propagation; position of the lens is shown by the white solid line. (b) The intensity distribution at the focal plane (c) The phase distribution at the surface of the lens.

a


b



e

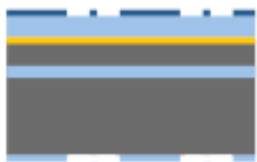

C
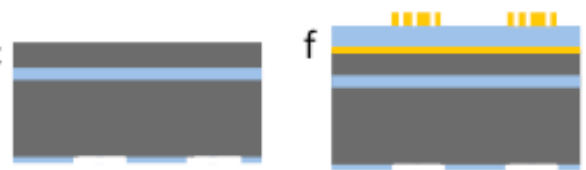

g
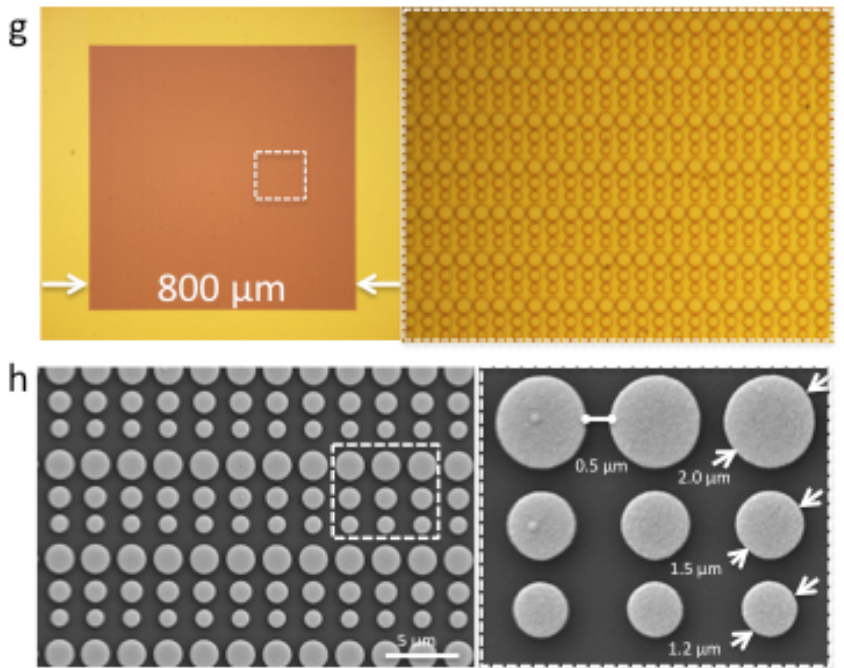

Figure 3. Fabrication of the planar lens. (a) - (f) Process flow of photolithography as descried in the section Fabrication: The flat lens. (g) Optical microscope image of the flat lens (h) Scanning electron microscope images of a part of the fabricated lens.

a $100 \mathrm{~nm}$ thick protective layer of silicon nitride $\left(\mathrm{Si}_{3} \mathrm{~N}_{4}\right)$ is deposited by plasma-enhanced chemical vapor deposition process. On the backside, a $110 \mathrm{~nm}$ thick layer of silicon dioxide $\left(\mathrm{SiO}_{2}\right)$ is deposited using the same process; this layer would serve as the oxide mask for etching the handle layer at a later stage of the fabrication. (b) A positive photoresist (SPR 700) is coated on the backside and exposed using a stepper tool (Autostep 200 i-line) to print circular windows, which would be perfectly aligned with the flat lenses to be fabricated on the topside. Using the developed photoresist as an etch-mask, the silicon dioxide layer is plasmaetched to produce the windows. (c) Next, the topside protective layer of $\mathrm{Si}_{3} \mathrm{~N}_{4}$ is cleared in hot phosphoric acid $\left(85 \% \mathrm{H}_{3} \mathrm{PO}_{4}\right.$ at $165{ }^{\circ} \mathrm{C}$ for 5 minutes). (d) Now we start fabricating the flat lens on the topside. We deposit $200 \mathrm{~nm}$ thick layer of gold (using electron beam vapor deposition), followed by $400 \mathrm{~nm}$ thick $\mathrm{SiO}_{2}$ (using PECVD). (e) We choose a bilayer resist for facilitating clean liftoff. The topside is coated with the photoresists LOR 3A followed by SPR 700. The resist bilayer is exposed using stepper, ensuring each lens structure is accurately aligned with the previously etched backside windows. (f) Finally, $50 \mathrm{~nm}$ gold with an adhesion layer of $5 \mathrm{~nm}$ thick titanium is deposited using e-beam vapor deposition, and lifted-off in remover-PG. This results in the gold disks constituting each lens. Figure $3 \mathrm{~g}$ shows the optical microscope image of one of such lenses. Figure $3 \mathrm{~h}$ shows the scanning electron microscope images of a section of the fabricated lens.

\section{Integrating flat lens with MEMS}

To integrate the flat lens with an external MEMS device, we try to "tear out" the lens from the thick SOI wafer. To do this, we start by etching the $600 \mu \mathrm{m}$ thick handle layer in xenon difluoride $\left(\mathrm{XeF}_{2}\right)$. Xenon difluoride etch is a highly selective, isotropic, dryetch process for silicon [8]. The backside window made with $\mathrm{SiO}_{2}$ provides the entry point for the xenon difluoride gas to react with the bulk of silicon in the handle layer. Due to excellent selectivity of $\mathrm{XeF}_{2}$ to silicon versus $\mathrm{SiO}_{2}$, the $200 \mathrm{~nm}$ thick buried-oxide layer of the SOI wafer also serves as the etch-stop layer. The etching process is visually inspected in-situ until all the silicon directly beneath the lens is etched out, and the lateral extent exceeds the outer dimension of the lens.



Figure 4. Optical microscope image after etching of the SOI handle layer from the backside and from the topside. 
Figure 4 shows optical image of the etched portion from the backside as well as from the topside. From the top, the shadow beneath the square lens structure indicates the area where silicon has been etched out. Figure 5a shows the schematic and the scanning electron microscope image of the different layers building up the lens. After the $\mathrm{XeF}_{2}$ etch, we are left with a membrane that is only $2.8 \mu \mathrm{m}$ in thickness and almost $1.35 \mathrm{~mm}$ in diameter (see Fig. 4).

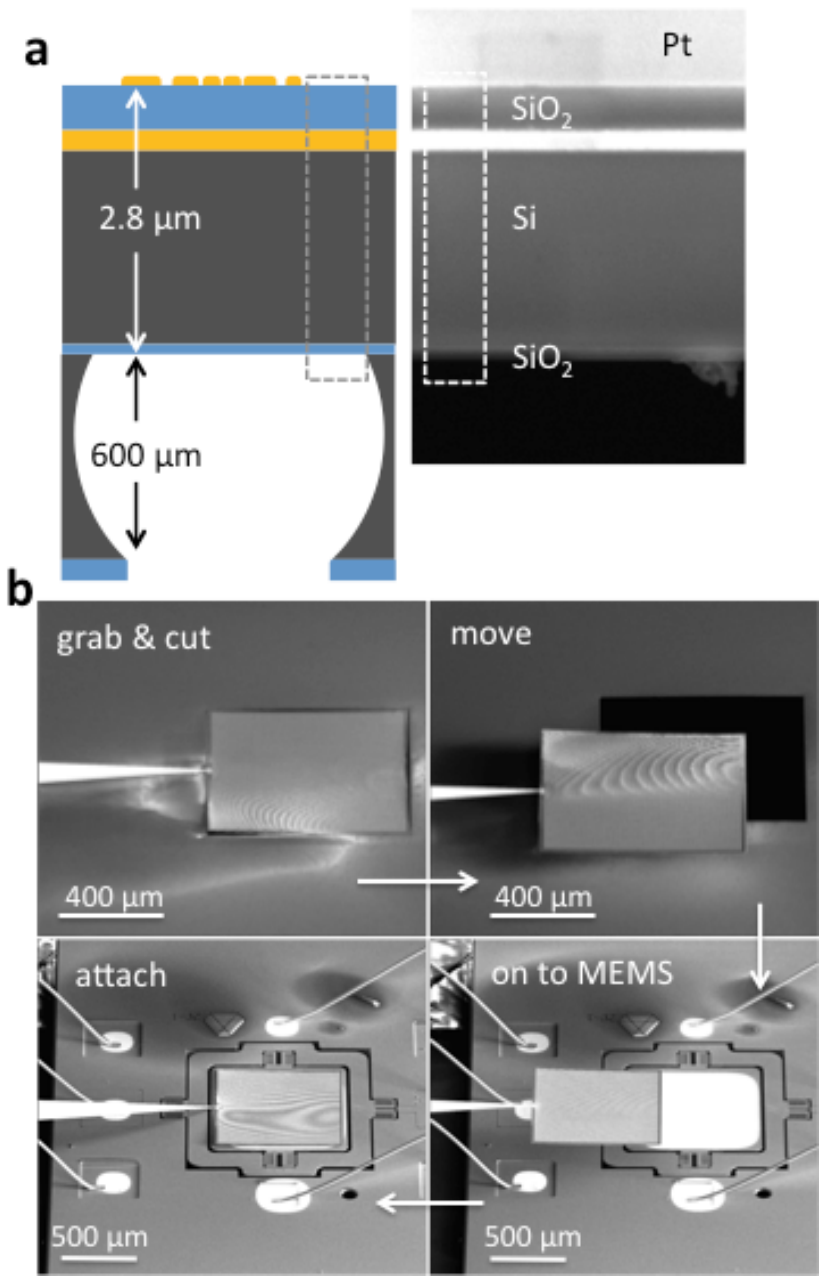

Figure 5. Integrating planar lens with MEMS. (a) Schematic of different constituting layers after etching of the SOI handle layer (left), and (right) SEM of the layers constituting the membrane on which the gold nano-discs are placed. (b) Stages of integration of the flat lens with an external MEMS platform.

For the next stage of fabrication, we use the focused ion beam (FIB) tool integrated with a micromanipulator needle to assemble the lens with the MEMS device. Figure $5 b$ depicts the steps of the process. We trace the focused ions around the periphery of the lens to cut out most of the structure, except a small portion. Here we weld the needle-tip of the micromanipulator by depositing platinum. Now the rest of the structure is released; the membrane is free from the surrounding solid substrate and is held only by the micromanipulator needle. We move the membrane out of the substrate and on to the MEMS platform by controlling the micromanipulator arm. After the lens is placed and aligned with the central platform of the MEMS, it is glued or welded in small patches with ion-beam assisted deposition of platinum. Finally the needle is cut away by milling with focused ion beam.

\section{EXPERIMENTAL CHARACTERIZATION}

\section{Optical measurement}

The optical performance of the lens is measured before integration onto the MEMS actuator, while it is still on the solid SOI wafer. To measure the focusing performance of the lens, we use a quantum cascade laser at $4.58 \mu \mathrm{m}$.

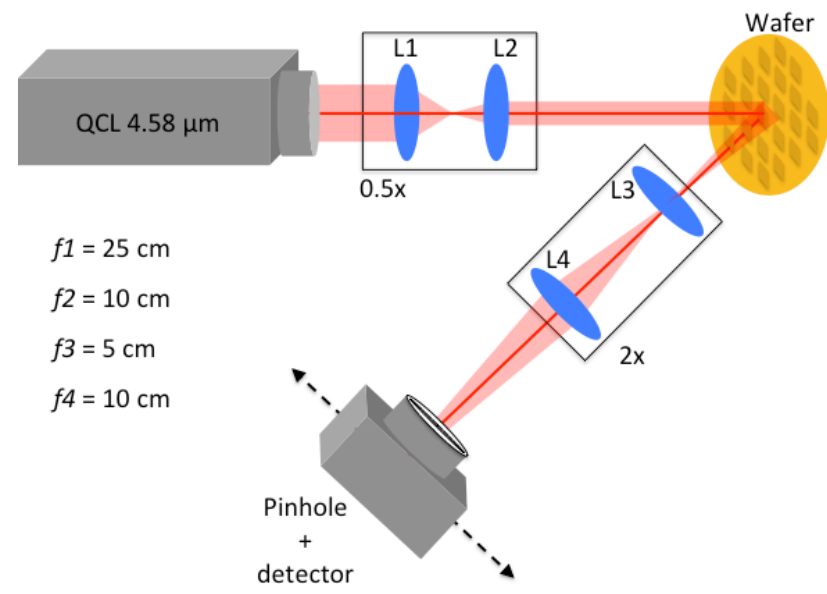

Figure 6. Schematic showing experimental arrangement used for characterization of the optical response of a flat lens.

Figure 6 shows the schematic of the experimental arrangement. The laser beam is downsized by 0.5 times, such that the incident beam is only slightly larger than the $800 \mu \mathrm{m}$ square lens. The light is incident normal to the lens surface. The lens reflects the incident beam at $45^{\circ}$ to the incoming path, and focuses the light at a distance of $2 \mathrm{~mm}$ away from its surface. The focused light is magnified two times by the lens pair L3 and L4 (see Fig. 6) and the intensity is detected by a thermo-electrically cooled mid-IR detector through a $10 \mu \mathrm{m}$ pinhole.
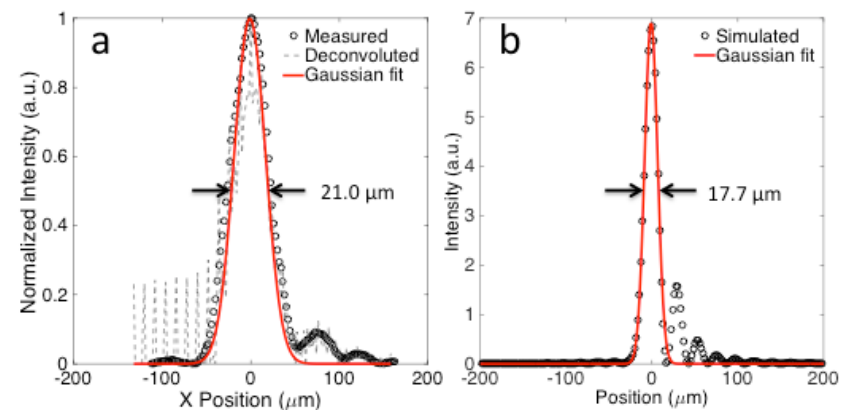

Figure 7. Focusing characteristics of the lens. (a) Experimental and (b) Simulated intensity profile measured across the focal line.

The detector-arm assembly is mounted on a rotating stage centered about the incident point on the wafer. This ensures we can precisely rotate the arm to detect the reflected light at an angle of $45^{\circ}$ to the incoming beam. The telescope (lens L3 and L4) and the detector can separately move along the reflection path so that all the components can be placed to accurately capture the intensity at the prescribed focal length of the lens. After the focal plane is detected, the detector is scanned linearly, across the reflection path, 
in $2 \mu \mathrm{m}$ steps. This gives us the intensity distribution across the focal line as shown in Fig. 7; the experimentally measured profile agrees well with the simulated result. The asymmetric distribution of low-intensity sidebands, in both the simulated and experimental result, is due to the $45^{\circ}$ phase ramp imposed on the lens surface to achieve the non-normal reflective focusing.

\section{Mechanical measurement}

To characterize the mechanical response of the MEMS device, we mount it on a dual in-line package. Figure 8a shows the optical microscope image of the flat lens integrated with 2D MEMS scanner; the inset shows a packaged device ready for electrostatic testing. As shown on Fig. 8a, the MEMS device has rotational degrees of freedom about two orthogonal axes: the inner gimbal axis and the outer gimbal axis respectively. The torsional movement of the MEMS platform is achieved by electrostatic actuation of capacitive vertical comb drives [9].

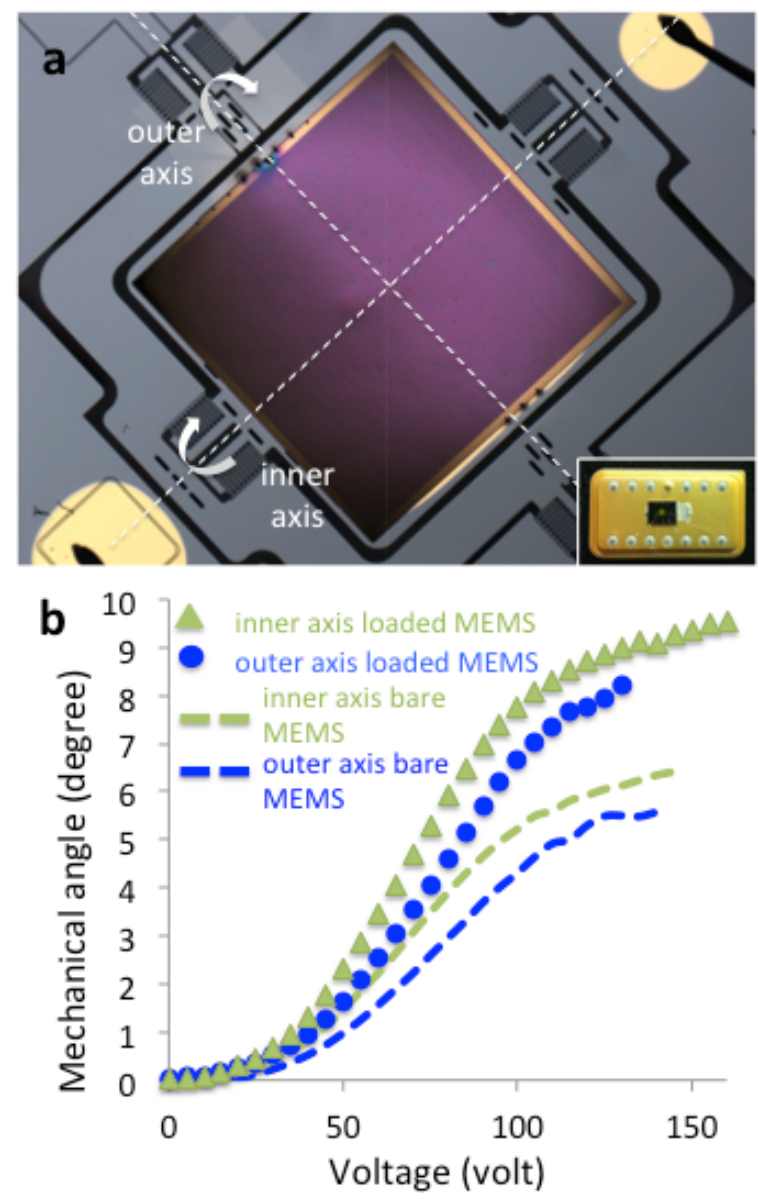

Figure 8. Mechanical characterization: (a) Optical microscope image of the flat lens integrated with MEMS; inset shows the device mounted on a dual in-line package ready for electrostatic actuation. (b) Electro-mechanical response of the MEMS platform with and without the flat lens.

Figure $8 \mathrm{~b}$ shows the mechanical response of the MEMS platform when a voltage is applied either across the inner or the outer axis. As the applied voltage is increased, the platform starts rotating until a saturating region is reached beyond which the comb drives cannot be moved further. We perform this experiment under an optical profilometer; for each applied voltage we capture an image of the MEMS surface. The tilt in the MEMS platform is calculated with respect to a rigid part of the peripheral structure. The measurements are taken for bare MEMS, i.e., before the flat lens has been integrated, and also for the final device loaded with the flat lens. As shown in Fig. 8b, even after the addition of the flat lens on the MEMS, the electromechanical response of the MEMS has not been compromised.

\section{CONCLUSION}

In summary, we have presented an electromechanically active focusing device in the form of a thin and flat surface. The planar optical device is designed for mid-IR wavelength and successfully integrated with a micro-electro-mechanical platform. The MEMS platform provides mechanical control of the flat lens up on electrostatic actuation. The lens can be tilted by $\sim 9$ degrees and hence the focal spot. We plan to test the optical characteristics of the lens while it is being actuated by the MEMS. Also, it would be interesting to study the high frequency scanning of the focal spot, while the MEMS is dynamically actuated. This proof-of-concept integration of flat optical devices with MEMS can be extended to visible and other parts of the electromagnetic spectrum implying potential for application across wider fields.

\section{ACKNOWLEDGEMENT}

Use of the facilities at the Center for Nanoscale Materials, Argonne National Laboratory was supported by the U.S. Department of Energy, Office of Science, Office of Basic Energy Sciences, under contract number DE-AC0206CH11357.

\section{REFERENCES}

[1] N. Yu and F. Capasso, "Flat optics with designer metasurfaces", Nature Materials 13 (2014).

[2] T. Roy, E. T. F. Rogers, and N. Zheludev, "Sub-wavelength focusing meta-lens", Optics Express, 21, 6 (2013).

[3] F. Aieta, P. Genevet, M. A. Kats, N. Yu, R. Blanchard, Z. Gaburro, F. Capasso, "Aberration-Free Ultrathin Flat Lenses and Axicons at Telecom Wavelengths Based on Plasmonic Metasurfaces", Nano Letters 12, 4932 (2012).

[4] T. Roy, A. E. Nikolaenko and E. T. F. Rogers, "A metadiffraction-grating for visible light", Journal of Optics 15,8 (2013).

[5] N. Yu, P. Genevet, F. Aieta, M. Kats, ...F. Capasso, "Flat Optics: Controlling Wavefronts with Optical Antenna Metasurfaces", IEEE Journal of Selected Topics in Quantum Electronics, 19, 3 (2013).

[6] L. Huang, X. Chen, H. Mühlenbernd, H. Zhang,... S. Zhang, "Three-dimensional optical holography using a plasmonic metasurface", Nature Communications, 4, 2808 (2013).

[7] X. Ni, A. V. Kildisev, V. M. Shalaev, "Metasurface holograms for visible light", Nature Communications 4 (2013)

[8] G. T. A. Kovacs, Micromachined Transducers Sourcebook, McGraw-Hill.

[9] I. W. Jung, S. Rattanavarin, P. Sarapukdee, M. J. Mandella, W. Piyawattanametha, and D. López, "2-D MEMS Scanner for Handheld Multispectral Confocal Microscopes", 2012 International Conference on Optical MEMS and Nanophotonics (OMN) IEEE (2012).

\section{CONTACT}

*Daniel Lopez; dlopez@,anl.gov 